\title{
Consistent churn of early career researchers: an analysis of turnover and replacement in the scientific workforce
}

\author{
Clara Boothby*1, Staša Milojević ${ }^{1}$, Vincent Larivière ${ }^{2.3}$, Filippo Radicchi ${ }^{1}$, Cassidy R. Sugimoto ${ }^{4}$ \\ ${ }^{1}$ Indiana University, Department of Informatics, Bloomington, IN (USA) \\ 2 École de bibliothéconomie et des sciences de l’information, Université de Montréal, Montréal, Québec (Canada) \\ 3 Observatoire des sciences et des technologies, Centre interuniversitaire de recherche sur la science et la \\ technologie, Université du Québec à Montréal, Montréal, Québec (Canada) \\ ${ }^{4}$ School of Public Policy, Georgia Institute of Technology, Atlanta, Georgia, USA. \\ * Corresponding Author, crboothb@iu.edu
}

Author Contributions: CB, SM, \& CS conceptualized the idea and motivation, VL supplied data; CB conducted data analysis and visualizations, wrote the manuscript; FR \& CB developed the model, SM,VL, FR \& CS contributed feedback and editing on the manuscript.

\begin{abstract}
Scientific advancement depends on sustaining a workforce trained in the scientific method. These scientists can in turn train the next generation of scientists and ensure that novel research continues to contribute to society. However, recent evidence shows that academic careers are shortening over time (Milojević et al., 2018). This coupled with a surplus of early career researchers (ECRs) relative to academic faculty positions raises the concern that talented scientists might not be retained in academic research. However, the empirical properties of the modern academic workforce are largely unknown. We use the publication histories of 3.5 million researchers to examine how career age composition of the scientific workforce has changed over 30 years. We find that for most fields there has been a relatively stable distribution of scientists at various career stages, with notable exceptions in Health and Physics. Using these data, we calculate the rate that researchers enter and depart the academic workforce, finding the most rapid turnover among ECRs. While varying the rates of turnover and replacement based on these observations, we develop a model and project a continuation of current career age proportions. This implies that the makeup of the workforce may remain stable with an abundance of ECRs. We conclude that the disconnect between the number of ECRs and the number of available long-term academic positions may be attributed to the collaborative nature of the academy. The stabilization of the workforce with a high proportion of ECRs urges a reassessment of how academic careers are portrayed.
\end{abstract}

Keywords: Academic Workforce, Turnover, Early Career Researchers, Training 


\section{Introduction}

Training new scientists is essential to ensure that the scientific workforce is fully staffed with talented researchers and able to make advancements. Since the foundation of the University of Berlin by Wilhelm von Humboldt in the early 19th Century, the doctoral degree is considered as the mechanism through which the next generation of researchers is trained. For established researchers, the mentorship of doctoral students allows for the reproduction of the scientific community, allowing the current population of established researchers to produce the next generation that will replace them as they retire [1-3]. If the reproduction of the scientific workforce were their sole motivation, tenured researchers would be expected to train one new scientists over their career. However, Larson et al. [1] estimate that the reproductive number for full professors on average is $7 \mathrm{PhD}$ researchers

Many commentators on the academic job market argue that the surplus of trained doctoral students is the result of institutional assumptions about the number of academic positions available $[4,5]$. However, an explanation that sees recruitment only as a mechanism to reproduce the tenured workforce may be overlooking the role of current early career researchers as a working population. Adding qualified team members to scientific endeavors may also be a way for researchers in advisory positions to increase the productivity of their research team [6]. The fact that researchers in training frequently fulfill crucial roles in the production of science may be a greater motivation for scientific training than the speculation that new researchers will be needed to replace retiring professors. Graduate students are very frequent collaborators for their advisors, as certain estimates show on average, each PI includes at least 1 student on $60 \%$ of their papers [7], and about a third of the total research output had some contribution by graduate students [8]. The division of labor in scientific working groups also often delegates essential research tasks, including lab maintenance, data collection, and analysis to $\mathrm{PhD}$ students and postdoctoral researchers [9-12]. Although increased specialization in the research lab creates a larger number of early career positions, recent graduates who performed these essential tasks may face greater challenges in securing a tenure track role [13]. Within the environment where there are more trained researchers than long-term positions, many researchers will face the choice of departing academia after only a short academic career or entering a series of short-term positions [14].

Against the backdrop of shortening academic careers, it is unclear why recruitment of students into scientific training continues to grow when, by many measures, there are too few long-term academic positions to accommodate the numbers of doctoral trained researchers $[4-5,15]$. Here, we use academic publishing data to examine the dynamics of entries and exits from the academic wing of the scientific workforce of the United States. We use these data to determine how the recruitment of new researchers into the academic workforce keeps pace with the rate needed to replace departing researchers, and the types of positions that newly trained researchers are most likely to fill. We also demonstrate that the current growth and replacement patterns of the academic workforce create a large share of early career researchers from the 1990 s to the present, and likely extending into the future. We aim to connect these concerns about the shortening of academic careers and over recruitment of new researchers to larger trends in the growth and turnover rates of the scientific academic workforce.

\section{Methods}

We obtained author lists from scientific publication data from the Web of Science (WOS), to which the author disambiguation algorithm from the Center for Science and Technology Studies (CWTS) at Leiden University was applied [25]. Data covers the years 1980 to 2019. As academic systems vary dramatically between countries, we focused only on the US scientific workforce and considered only papers for which 
there was at least one US affiliation. Since prior to 2008, there is no link between authors and their institutional addresses on papers, we considered as US researchers those for whom at least $85 \%$ of their papers have a US affiliation ( $N=3,525,183)$ (See Supplementary figure 3 ). However, for these researchers, the year of their first publication was retained and considered the beginning of the research career, regardless of whether this first paper was published with a US affiliation.

We approximate the end of the publishing career of a researcher as the year of the last publication of the researcher appearing in our dataset. We refer to the career ages of researchers at each year as a snapshot of the career age distribution. The most common issue in the current disambiguation method is splitting single authors [25], so to adjust for this, we filtered out authors who had only published one paper. The disciplinary classification used is that developed for the National Science Foundation, which categorizes each journal into one field and subfield [26]. We inferred the primary field for each researcher based on the broad subject category for the majority of their papers; in the case of ties, the first field to appear is assigned as the primary field because we are interested in the early career. Given that the dataset starts in 1980, researchers whose careers actually began before 1980 are incorrectly identified as starting their careers in the year 1980 or shortly afterwards. To correct for this, while the start of author publication histories is drawn from data between 1980 and 1989, the analysis period to begin in 1990, and researchers with career ages of 10 or more years are binned.

Researchers may also experience gaps in their publication histories. If these gaps occur near the start or end points of our dataset's timeframe, these gaps may be mis-inferred as researchers beginning or ending their careers, respectively (Supplemental Figure S2). For example, subset of researchers who appear to have a career age of 8 in 1990 (i.e. with their first publication in 1982) were likely publishing before 1980. To account for this, we also empirically calculated the probability that academic publishing histories continued after gaps of various lengths in each field (Supplemental Figure S3). We then adjusted our estimated counts of researchers at each career ages by multiplying the raw counts of researchers beginning their career in the years shortly after the start of the data set in 1980 by the appropriate probability that the apparent first publication was preceded by a gap.

We recognize that using the length of the publication career as a proxy for career age may limit our findings as there are differences in publication patterns between fields. For example, when researchers typically publish their first paper may differ between fields. We also acknowledge that researchers are publishing prior to earning their PhD more frequently now than in the past [19]. In addition, the exclusion of researchers with only one publication may cause an underestimation of attrition rates. However, as all of these limitations should result in an underestimation of the extent to which scientific careers have been shortening, we feel confident that the interpretation of our results remains valid

\section{Results}

\section{Consistent growth in science and the replacement rate}

Using publication data from 1990 to 2010 to measure career ages of scientific researchers we find that for each year the total numbers of researchers entering and leaving the academic workforce follows a positive linear relationship (Fig 1A) that explains 91\% of the variance for the aggregated, All Fields scientific workforce (Supplementary Table 1). We do observe differences in the strength of this relationship between scientific fields. For many fields, such as Health, Chemistry, Earth \& Space, and Science \& Technology, we observe a strong correlation (maximum correlation coefficient of $97 \%$ in Health) indicating a linkage in the number of researchers entering and leaving the field, such that both entries and 
departures grow in tandem over time at a steady rate. However, in some fields, such as Physics with a correlation coefficient of $36 \%$, there is considerably more variance indicating less consistent growth in the workforce over time. The consistency of the overall relationship of researchers entering and leaving the workforce suggests that the entry of researchers in training is offset by a proportionate number of researchers leaving academic environments. This relationship between entries and exits may be suggestive of a balance, it's also a sign that there is not enough space in academic research to absorb the rate of recruitment.

We identify two parameters, turnover and replacement rate, that together may describe the overall growth of the academic workforce. We define the turnover rate as the proportion of researchers in the academic workforce to publish their last paper in a given year; in other words, the proportion of the academic workforce whose roles will open in the following year, a figure that we calculate has a mean of $11 \%$ per year. We also define the replacement rate as the ratio of researchers entering to that of those leaving the US scientific workforce each year, such that a replacement rate of 1 would represent a state of equilibrium where the number of entering researchers exactly matches the number of departing researchers and the workforce remains the same size. We find the mean replacement rate in the scientific workforce in the United States between 1990 and 2010 to be 1.26, meaning on average more researchers enter the workforce each year than leave. Both turnover $(T)$ and the replacement rate $(R)$ can be used to predict the overall growth rate of the workforce:

\section{Equation 1. $\mathrm{G}=\mathrm{T} \cdot(\mathrm{R}-1)$}

Where $\mathrm{G}$ is the compound annual growth rate of academic science. In this way, increasing the turnover and replacement rate each have the potential to increase the overall workforce growth because increasing the turnover also increases the number of open positions that will be filled by a replacement rate that is usually higher than the equilibrium point of 1 . Albeit, when the replacement rate dips below 1 , then the size of the workforce in the following year would be smaller, but this is rarely the case (Supplemental Figure S6).

We also find field differences in both the replacement rates and turnovers, which may indicate different behavior regarding growth and composition workforce composition between fields (Figure 1B-D). Across fields, Earth \& Space and Chemistry seem to represent the extremes of both mean replacement rate and mean turnover, with Chemistry having the highest turnover (0.13) and lowest replacement rate (1.18), while Earth \& Space has the reverse, with a low turnover (0.07) and high mean replacement rate (1.48) (Fig 1B). The lower turnover indicates a greater retention of currently working researchers in Earth \& Space than in Chemistry, and the higher replacement rate means that a larger number of entering researchers fill the roles vacated by departing researchers in Earth \& Space. Despite these opposite extremes, each field's growth is comparatively similar, around 0.02 in Chemistry and 0.03 in Earth \& Space. In fact, most other fields, which the exception of Health, occupy a similar band of growth rates ranging from $0.02-0.04$, even as their replacement rates and turnovers vary. When the same growth rate is maintained, there appears to be an trade-off between turnover and replacement rate, but in Health, it is also possible for both a high replacement rate (1.48) and a high turnover (0.11) to result in a higher growth rate of around 0.05 . 

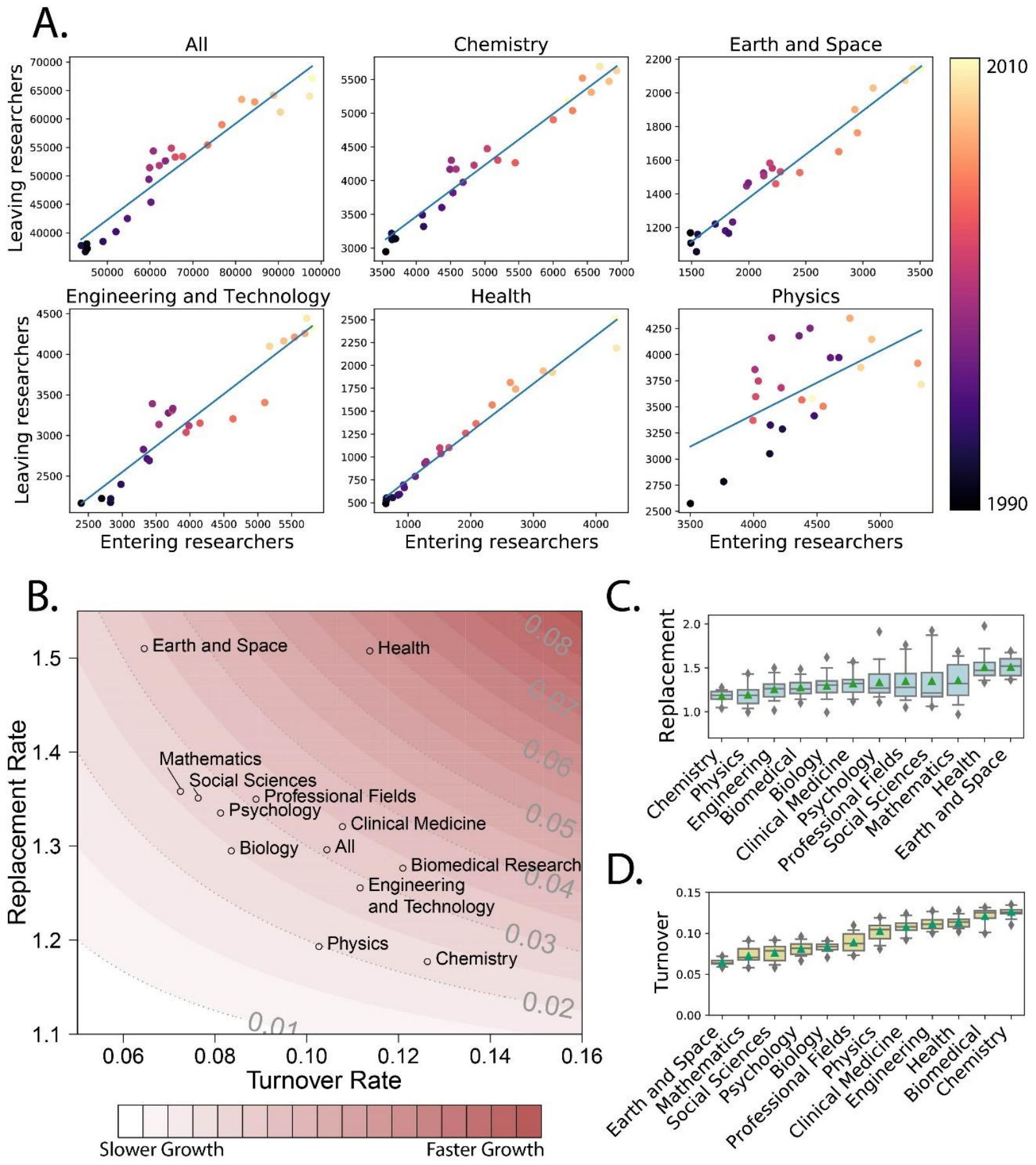

Figure 1. (A) The relationship between the number of researchers who have published their first publication and their last publication in a given year for the full academic workforce, and 5 separate fields: Chemistry, Earth and Space, Engineering and Technology, Health, and Physics. Solid lines indicate a linear regression. (B) The theoretically predicted growth rate given turnover and replacement rate; darker shades of red represent faster growth. The mean turnover and mean replacement rate situate each field, predicting their growth rate (See Supplemental Figure S8 for model fits for each field). (C) The distributions of replacement rates over time for each field. (D) The distributions of turnovers over time for each. 
If we understand the modest growth rates of most fields to be indicative of constraints on growth that fields experience through funding, facilities, or other limitations, the apparent trade-off between turnover and replacement may represent different strategies that fields use in decisions about recruiting, training, and retaining personnel. Fields with high turnovers and low replacement rates, like Chemistry, Engineering \& Technology, Biomedical Research, and Physics, accept the entry of a larger proportion of new researchers in comparison to their size. By doing this they may be maintaining a modest growth rate by keeping their replacement ratio closer to 1 . On the other hand, fields with high replacement rates and low turnovers, like Mathematics, Social Sciences, Professional Fields, Psychology, and the extreme case of Earth \& Space, may replace a smaller group of departing researchers with an entering cohort that is larger in relation to the number of departing researchers. Beyond maintaining growth in scientific fields, these strategies may result in different outcomes in graduate training. Lower turnovers in a field may indicate a greater emphasis on retention in that field or slower recruitment, which may allow for a higher chance of a long-term career within that field. Increased chances of a long-term career may justify a greater outlay of resources during training and allow research to be less disrupted by frequent personnel changes. However, smaller turnover rates also allow less availability for new researchers to enter the field and complete training; fields that require many trained graduates, particularly when suitable opportunities exist for graduates outside the academic sphere, may instead find greater benefit in turning over early career researchers at a higher rate to accommodate larger numbers of prospective recruits.

\section{Stability in the Career Age Distribution of the Academic workforce}

Over all fields of scientific research over time, the proportion of researchers in each career stage follows a relatively stable distribution, with little yearly fluctuation in the proportional size of each career age (Fig 2). Early career researchers in the first few years occupy a large proportion of the academic workforce, as $50 \%$ of all researchers are career age 5 or younger. Each career age before the $10+$ senior researcher category occupies a successively smaller proportion of the academic workforce as career age increases. This tapering in the proportions of researchers at higher career ages is expected, as the size of each successive career age is governed by the size of the earlier career age in the year prior to it. For example, if there are 200 researchers at career age 2, in the next year, the size of the workforce in the successive career age can only be the same size or smaller than the group in the previous year. We find that the group of senior researchers of career ages 10 and greater comprise about $35 \%$ of the academic workforce.

This distribution is considerably robust, exhibiting only very gradual change over time from 1980 to 2010 . For the aggregate of all fields (Fig 2, top left panel), the proportions of early career ages exhibit very gradual increases over the period from 1990-2010, but these increases are small enough that the significance of these increases is inconclusive (See Supplemental Table 2). This stasis in the career age distribution for the aggregated academic workforce, as well as the fields Chemistry, Earth \& Space, and Engineering \& Technology indicates a remarkable capacity for academic fields to settle on an equilibrium that creates a consistent proportion of researchers at each career level in their field from year to year. From a labor perspective, work within an academic field necessitates the very distribution that has naturally arisen in the proportions of researchers at each career level over time.

However, in certain fields there are still notable changes in the proportions of career ages over time, particularly for fields experiencing high or low rates of growth. In Health, which exhibits the highest growth rate of any field (Supplemental Table 3), we find gradual increases in the share of early career researchers over time, with the earliest career stages exhibiting the highest gains, but with corresponding decreases in the proportions of senior researchers. In other words, while Health grows as a field, most of these expansions are taking place in the roles available to early career researchers as opposed to in 
positions with greater capacity for longevity. On the other hand, Physics before 2000 shows a gradual increase in the proportion of researchers in the 10+ group against a small decrease in the proportion of early career researchers. As Health grows younger as a field while it grows in size, Physics seems to have admitted fewer new researchers during the period when its growth was slowed, resulting in a comparatively older workforce. These resulting differences between career age distributions likely reflect the kinds of labor distributions that these fields require: In fields that require a greater amount of experienced supervision, the retention of senior researchers to oversee the work and training of early career researchers may require a larger ratio of senior to junior researchers. In contrast, Health's growing ranks of early career researchers may be serving a research style that requires a lot of labor, but fewer senior researchers to train and supervise.
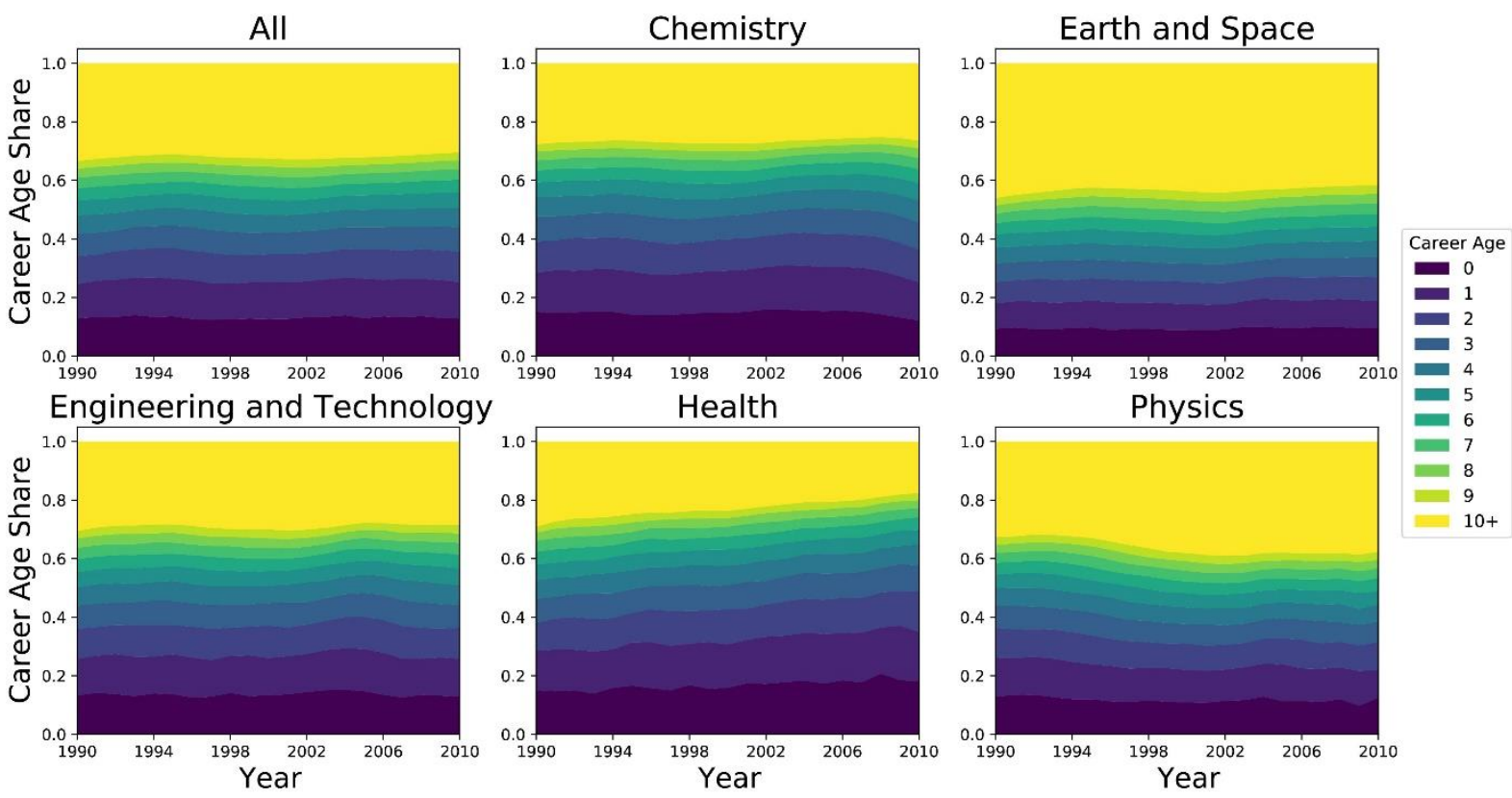

Figure 2. The proportion of researchers at each career age (years) in each year for the scientific workforce in aggregate (top left), and by field for Chemistry, Earth \& Space, Engineering \& Technology, Health, and Physics.

\section{Turnover decreases for later career ages}

As the entry of new researchers is balanced against the departure of existing researchers, we next investigated the career ages of departing researchers using turnover: the proportion of the total workforce that has their last publication in a given year. With a mean turnover rate of 0.10 per year for all fields, it would theoretically be possible for the entirety of the academic workforce to be replaced every 10 years. However, the fact that researchers may leave at any career age allows careers to be longer or shorter than that. Beginning after the first full year since writing their first paper, we observe a high rate of departure rate within earliest career academic positions, with decreasing proportions of departing researchers in each successive career age (Fig $3 \mathrm{~d}$ ). In this way, for each successive year that a researcher spends working and publishing within the academic workforce increases the probability that they will continue publishing in academic science. Turnover rates are not uniform across the different career age populations in academic science, and that the positions with the fastest turnover are those occupied by those in the very earliest stages of their careers. The higher turnover for early career ages further emphasizes that the stability of career age proportions does not mean that researchers have stability in terms of career longevity. 
These proportions are largely consistent in that later career ages consistently have lower turnovers than earlier career ages in each year, but we do observe a possible increase in turnover rates over the mid1990s until a high water mark in 1997 followed by a decrease up to the end of the analysis period in 2010 .Over a career in which an individual must pass through each career age without ceasing publication, any small reduction in the probability of attrition could additively increase the chances of a long-term career. The reduction in the turnover between 1997 and 2010 could indicate an increased emphasis of retaining early career researchers past their first, most vulnerable years.
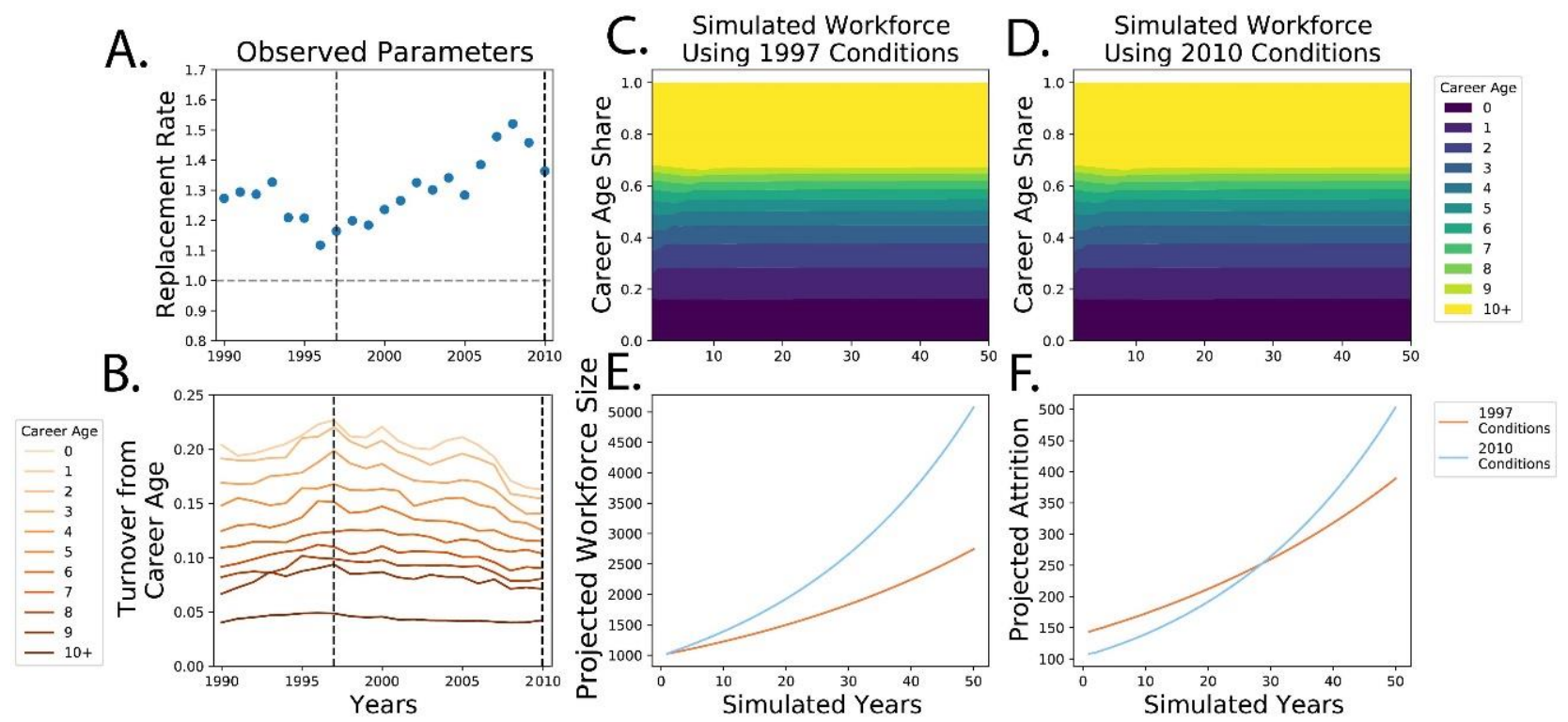

Figure 3. (A) The yearly replacement rate for all fields over time. Horizontal dashed line indicates equilibrium for the replacement rate. Vertical dashed lines indicate the replacement rates that were used for the projected workforce conditions in 1997 and 2010 respectively, (B) The proportion of each career age group across all fields that publishes their last paper in each year (Turnover). Vertical dashed lines indicate the turnover rates that were used for the projected workforce conditions in 1997 and 2010 respectively (C-D) The simulated career age distribution based on the replacement rate and turnover rates in the years 1997 and 2010 respectively. The starting values of the model rely on the mean proportion of researchers at each career age in each year. See Supplementary materials for the full description of the model. (E) The projected size of the workforce from a starting workforce size of 1000 if the turnover and replacement rates in 1997 and 2010 remain constant. (F) The projected attrition from a starting workforce size of 1000 if the if the turnover and replacement rates in 1997 and 2010 remain constant.

Since the decreases in turnover for all career ages coupled with an increase in replacement rate may be the current modern trend in the academic workforce, we used the career age specific turnover profiles from 1997, the high water mark for career age turnover, and 2010, the last year of analysis, in simulations of the dynamics of career age in the academic workforce (See Supplementary Equations 1-3). Each simulation starts at the actual career age distribution of the scientific workforce for the year in which the turnover profile was pulled, 1997 and 2010 respectively, but with a total workforce size of 1000 researchers. Although the conditions for 1997 and 2010 have different turnover and replacement rates, each simulation resulted in a career age distribution that exhibits very little change in the proportion of each career age over time. While decreasing turnover alone results in a gradual reduction in the share of early career researchers over time (Supplementary Figure 11), the decrease in turnover between 1997 and 2010 seem to have been counter-weighted by the increase in replacement rate. As different realworld sets of parameters result in similar stable states, it may illustrate the strong tendency of the 
academic workforce to seek a kind of equilibrium with a desirable distribution of junior and senior researchers.

While there is stability in the proportions of researchers at each career age, when projected over the next 50 years, the turnover and replacement rates of 2010 result in a more rapid rate of growth and ultimately higher rates of attrition than 1997 conditions. While the lower turnover rates in 2010 initially result in a lower number of researchers leaving the academic workforce, in the long run, the conditions in 2010 will surpass the attrition rate projected by the 1997 conditions. In this simulation, the initial reduction in the attrition can be credited to the reduced turnover in the $\mathbf{2 0 1 0}$ model. However, as the simulation results in a higher growth rate, it suggests that accelerating growth in the size of the academic workforce can nonetheless result in higher attrition, regardless of lower turnover. In this way, while focusing on retention as a means to counter high attrition may show promise in the short term, the source of skyrocketing attrition rates in academic research may ultimately lie in the overall growth in size of the academic workforce. Even if a larger proportion of researchers are retained in longer careers, as the recruitment of academic scientists maintains pace with the demands for early career researchers, the number of researchers leaving academic research may necessarily continue to rise.

\section{Discussion}

Using researchers' range of publication dates, we have calculated the career age of the academic workforce for each year from 1990 to 2010. Although there is a higher number of researchers entering science each year, we find that there are commensurate increases in the number of researchers who leave academic science each year. Relating the entry of new researchers to the departure of existing researchers stands in contrast to previous reproductive models, which associate the entry of new researchers to those that senior faculty can train. We also find that an average of $10 \%$ of researchers leave academic science each year, and that these departing researchers tend to be in the very early career stages.

A frequent interpretation of the decreasing availability of long-term academic positions is that there is an oversupply of PhDs trained for the number of available tenured positions $[15,16]$. These interpretations often rest on the assumption that tenured professors train students in order to fill similar tenure-track positions. However, Milojevic et al. [17] have found that, as research careers in academia shorten, there is a population of researchers whose role as active publishing researchers may be characterized as temporary. Our finding that most departures from publishing basic research occur in the early career support the interpretation that the entry of scientists into PhD training may serve the shorter term goal of filling early career positions on research teams or training researchers for positions outside academia rather than the longer term goal of reproducing tenured faculty. The median time to complete a PhD from starting a graduate program for all fields is 7.3 years from the start of graduate school and 5.6 from starting a doctoral program specifically [18]. While the typical timing of first publications in relation to doctoral matriculation varies between fields and individuals [19], the high probability of leaving the academic workforce in the earliest career ages suggests that many researchers will not publish after they have completed graduate education. For early career researchers, a greater emphasis on training rather than publication may be necessary to support transition to fulfilling careers, both inside or outside academic science. If academic research requires these large shares of junior researchers in the current production of scientific research, we also urge a re-imagining of these roles traditionally fulfilled early career researchers, so that they tie more directly to training, or they are performed by researchers in more stable long-term positions than junior scientists concurrently completing academic training. 
We also find that the career age distribution of the academic workforce may gradually be shifting toward a younger workforce in fast-growing fields like Health. Furthermore, our modeling demonstrates that the higher turnover rates for early career researchers contributes to the higher representation of early career researchers in the academic workforce. Previous research has uncovered indicators that the tenured academic workforce is aging [20], but these conclusions could also benefit from accounting for the large share of early career groups in the larger academic workforce. Because every successive year a researcher remains in academia reduces the probability of leaving it, researchers, experienced Pls are still able to maintain successful long-term research careers as early career researchers turn over faster and grow more populous around them.

In aggregate for all fields, as the career age distribution shows only very slow, ambiguous growth in the early career ages, the picture is less clear. We suspect that current frustrations about the academic job market may be rooted in conditions of the academic workforce that are neither new nor the effects of recent policy decisions. As career age turnover rates have shown a small decrease between 1995 and 2010, our model may indicate that a small increase in the proportion of long-term researchers is possible if the turnover rates remain low. However, lower turnover rates also come with trade-offs: lower turnover rates may leave less capacity for new researchers to enter academic science and lower availability of graduate education. Because no field in science has unlimited resources, changes in growth patterns, recruitment, and retention will necessarily have an effect on the other factors at play governing field sizes and growth. Furthermore, another complicating factor towards the stability of the career age distribution is that career age describes the level of their experience in academic research, but not the position that the researchers occupy. As tenured and tenure-track positions are being replaced with temporary positions [20], so while the career age distribution may appear relatively stable, it is also possible that academic employment may be less stable than in the past.

In addition, high turnover rates within academic science are not reflective of an exodus of trained researchers from science because ample opportunities for scientific research are present outside academia as well. In fact, we find that the fields with the higher rates of turnover (Chemistry, Engineering and Technology, and Biomedical research) are also fields with strong ties to industry, and the high turnover rates here suggest that scientists in these fields frequently pursue industry positions after their training period. In this way, fields with a high probability of short careers among their participants could be interpreted as industry feeders. Surveys of student preferences for entering industry indicate field differences in the attractiveness of industry and other nonacademic positions; Chemistry for example has been shown to have a higher percentage of its students rating an industry career as very attractive, with as high as $37 \%$ and $17 \%$ of researchers rating work at established and start up industry firms as extremely attractive [22-23]. Researchers in industry are still active participants in scientific research, and with near $40 \%$ of doctorates entering large industry research firms by some estimates, industry hiring has been presented as a reliable way to transfer expertise from academia to the greater society [24]. As we have shown that reductions in attrition are often linked to reductions in the recruitment of new researchers, any policy interventions to reduce attrition by decreasing turnover should be mindful not to reduce the capacity to provide graduate education to scientists planning to enter non-academic fields.

Voluntary departure for research opportunities in the government or private sector are common reasons for PhD recipients to leave academic research $[18,23]$. However, this does not invalidate the fact that many PhD trained researchers who desire to pursue long-term academic careers are stymied by a low availability of long-term tenure track positions $[4,5]$. Despite these factors, long term academic positions are still frequently framed by advisors as the preferred outcome of PhD training [22-23]. We agree that the narratives of involuntary ends to research careers due to constraints at not finding academic positions 
are pervasive and the lived experience of individual researchers. Our point to supplement and contextualize these narratives is that within the larger context of the scientific workforce, the positions fulfilled by early career researchers were structurally short-term. These expectations and the nature of $\mathrm{PhD}$ training may need to be adjusted to reflect the reality that large proportions of scientists who participate in academic publishing during their training periods will not ultimately pursue long-term careers within academic science.

\section{Acknowledgments}

We would like to thank the Sugimoto-Larivière joint team meeting group and the Science Genome team at Indiana University for their helpful comments and engagements during meeting presentations. This material is based upon work supported by the Air Force Office of Scientific Research under award number FA9550-19-1-0391.

\section{References}

1. R. C. Larson, N. Ghaffarzadegan, \& Y. Xue, Too Many PhD Graduates or Too Few Academic Job Openings: The Basic Reproductive Number R0 in Academia. Syst. Res. Behav. Sci. 31,6, 745-750. (2014).

2. R. D. Malmgren, J. M. Ottino, \& L. A. Nunes Amaral, The role of mentorship in protégé performance. Nature 465, 7298, 622-626. (2010)

3. J. F. Liénard, T. Achakulvisut, D. E. Acuna, \& S. V. David. Intellectual synthesis in mentorship determines success in academic careers. Nat. Commun. 9,1, 4840. (2018).

4. H. R. Bourne, A fair deal for PhD students and postdocs. Elife, 2, e01139. (2013)

5. E. Perlstein, Generation Postdocalypse. Trade Secrets: A Blog from Nature Biotechnology. http://blogs.nature.com/tradesecrets/2014/07/18/generation-postdocalypse. (2014, July 18)

6. S. Milojević, Principles of scientific research team formation and evolution. Proc. Natl. Acad. Sci. U.S.A. 111,11, 3984. (2014).

7. B. Bozeman, \& J. Youtie, The Strength in Numbers: The New Science of Team Science. (Princeton University Press, 2017).

8. V. Larivière, On the shoulders of students? The contribution of $\mathrm{PhD}$ students to the advancement of knowledge. Scientometrics 90,2, 463-481. (2012).

9. K. Holden, Lamenting the Golden Age: Love, Labour and Loss in the Collective Memory of Scientists. Sci Cult (lond) 24, 1, 24-45. (2015).

10. V. Larivière, et. al., Contributorship and division of labor in knowledge production, Soc. Stud. Sci. 46, 3, 417-435. (2016).

11. N. Robinson-Garcia, R. Costas, C. R. Sugimoto, V. Larivière, \& G. F. Nane, Task specialization across research careers. ELife 9, e60586. (2020).

12. J. P. Walsh, \& Y. N. Lee, The bureaucratization of science. Res. Policy. 44, 8, 1584-1600. (2015).

13. E. Leahey, B. Keith, \& J. Crockett, Specialization and promotion in an academic discipline. Res. Soc. Stratif. Mobil. 28, 2, 135-155. (2010).

14. S. D. Cheng, (2021). What's Another Year? The Lengthening Training and Career Paths of Scientists. [working paper](2021) https://scholar.harvard.edu/files/sdcheng/files/ sdcheng_careers_paper.pdf (accessed 16 February 2022)

15. D. Cyranoski, N. Gilbert, H. Ledford, A. Nayar, \& M. Yahia, Education: The PhD factory. Nature 472, 7343, 276-279. (2011). 
16. D. Cuthbert, \& T. Molla, PhD crisis discourse: A critical approach to the framing of the problem and some Australian 'solutions.' Higher Education, 69, 1, 33-53. (2015).

17. S. Milojević, F. Radicchi, \& J. P. Walsh, Changing demographics of scientific careers: The rise of the temporary workforce. Proc. Natl. Acad. Sci. U.S.A. 115, 50. (2018).

18. Doctorate Recipients from U.S. Universities: 2018 (No. 20-301; Special Report NSF). National Science Foundation, National Center for Science and Engineering Statistics. (2019). https://ncses.nsf.gov/pubs/nsf20301/ (Accessed 16 February 2022)

19. C. J. F. Waaijer, B. Macaluso, C. R. Sugimoto, \& V. Larivière, Stability and Longevity in the Publication Careers of U.S. Doctorate Recipients. PLoS One 11, 4, e0154741. (2016).

20. J. Kaiser, The Graying of NIH Research. Science 322, 5903 , 848. (2008).

21. N. Fetcher, M. E. Lam, C. R. Cid, \& T. Mourad, Contingent faculty in ecology and STEM: an uneven landscape of challenges for higher education. Ecosphere, 10, 12, e02964. (2019).

22. H. Sauermann, \& M. Roach, Science PhD Career Preferences: Levels, Changes, and Advisor Encouragement. PLoS One 7(5), e36307. (2012).

23. M. Roach, \& H. Sauermann, A taste for science? PhD scientists' academic orientation and selfselection into research careers in industry. Res. Policy. 39, 3, 422-434. (2010).

24. N. Zolas, et. al., Wrapping it up in a person: Examining employment and earnings outcomes for Ph.D. recipients. Science, 350, 6266, 1367-1371. (2015).

25. E. Caron, \& N. J. Van Eck, "Large scale author name disambiguation using rule-based scoring and clustering." Proceedings of the 19th International Conference on Science and Technology Indicators, (2014). 79-86.

26. K. Hamilton, Subfield and level classification of journals (CHI Report No. 2012-R). Cherry Hill, NJ: CHI Research (2003). 